Research Article

\title{
Herd Behavior and Financial Crashes: An Interacting Particle System Approach
}

\author{
Vincenzo Crescimanna and Luca Di Persio \\ Department of Computer Science, University of Verona, Strada le Grazie 15, 37134 Verona, Italy \\ Correspondence should be addressed to Luca Di Persio; dipersioluca@gmail.com
}

Received 19 October 2015; Accepted 11 January 2016

Academic Editor: Yonghui Sun

Copyright ( 2016 V. Crescimanna and L. Di Persio. This is an open access article distributed under the Creative Commons Attribution License, which permits unrestricted use, distribution, and reproduction in any medium, provided the original work is properly cited.

\begin{abstract}
We provide an approach based on a modification of the Ising model to describe the dynamics of stock markets. Our model incorporates three different factors: imitation, the impact of external news, and private information; moreover, it is characterized by coupling coefficients, static in time, but not identical for each agent. By analogy with physical models, we consider the temperature parameter of the system, assuming that it evolves with memory of the past, hence considering how former news influences realized market returns. We show that a standard Ising potential assumption is not sufficient to reproduce the stylized facts characterizing financial markets; this is because it assigns low probabilities to rare events. Hence, we study a variation of the previous setting providing, also by concrete computations, new insights and improvements.
\end{abstract}

\section{Introduction}

Empirically studying the fluctuations in the dynamics of prices for various financial assets, it has been shown that related stock returns are distributed similarly to a Gaussian random variable, but with fat tails. The latter is due to the possibilities of having large fluctuations of the prices as it has happened, for example, during recent world financial crisis; see, for example, [1-3]. One possible theoretical explanation for such phenomena relies on the so-called econophysics approach which states that significant fluctuations in prices correspond to collective phenomena, such as crowd effects or herd behavior; see, for example, [4], [5], [6], respectively. The main purpose of the present work is to extend those approaches which have been based on the Ising model, as, for example, in [7-9], by defining a model characterized by $C$-potentials, such that its behavior rule, or rule of advancement, can be described by a dynamic of the Glauber-type. We notice that the latter approach allows us to build models which are easier to extend to more complex scenarios, for example, hierarchical lattice, that are the subjects of our different work, currently in progress.
In particular, our approach allows us to show that the existence of herd behavior among participants causes the fat tails property characterizing the distributions of real log prices. The goodness of the proposed model is analyzed by means of simulations where the stock market evolution is determined by a set of interacting particles, each of which represents a financial agent playing in the considered economic framework. We assume that the interactions between the aforementioned agents are driven by a potential $\Phi=\Phi(\Lambda)$, which is a measure of the role played by the agents in the set $\Lambda$. Following the idea developed in $[10,11]$, we will consider the temperature of the system as a parameter describing quantities related to the probability of possible crashes. In particular we are interested in those dynamics which admit phase transitions. In fact, we can relate latter phenomenon, which is a well-defined statistical-mechanics concept, with the excess of demand represented by heavy tails. Therefore, as it happens when a physical system reaches a critical point, in financial terms we have that the system crashes if it experiences an excess of financial movements along the same direction; namely, a large number of single agents buy or liquidate their financial position simultaneously. The latter phenomenon 
happens in correspondence with a critical temperature value $T_{C}$, giving rise to a phase transition.

The paper is organized as follows: in Section 2, we briefly outline the main characteristics of the so-called econophysics approach to the financial markets recalling two of the most relevant models used to describe the herd behavior in stock markets, namely, the Cont-Bouchaud model (see [5]) and the Sornette-Zhou model (see [8]); in Section 3, we describe our proposal, starting from the standard Ising potentials; in Section 4 we show our main theoretical results, and we also provide related numerical simulations for particularly relevant choices of the involved parameters; eventually, in Section 5, we give a résumé of the obtained results and we outline further lines of possible improvements and researches.

\section{The Econophysics Approach}

During the last decades several models belonging to the statistical-mechanics world have been used to describe the herd effect, namely, the phenomenon which arises when a group of individuals act collectively without a centralized direction. In what follows we briefly recall the main properties of two of the most relevant approaches exploited to describe the latter type of behavior within typical financial arenas, namely, the Cont-Bouchaud and Sornette-Zhou models; see [5], [8], respectively.

The model developed by Cont and Bouchaud is based on the idea that the herd effect, which has been identified as one of the peculiar characteristics of stock markets as well as one of the main causes of crashes, can be described thinking that the financial system is constituted by random clusters of agents who imitate each other. Moreover, different clusters are allowed to take decisions independently of each other. The latter random communication structure can be described exploiting the Erdös-Rényi graph theory: see, for example, $[12,13]$, where with each node/agent $i$ is associated a random variable $\sigma_{i}$ which can take the three following values: $+1,-1,0$, namely, the possible agent actions. In particular, the values $+1,-1$, and 0 , respectively, correspond to selling a stock, buying a stock, and to not trade at all. Concerning the edges of the graph, we have that each agent has the same probability $p \in(0,1)$, to be linked to another one. Such a framework can be also represented by using a potential function which admits a Gibbs measure, as shown in, for example, [14]. The latter Gibbs measure, for $p \simeq 1 / N, N$ being the number of agents, realizes the desired shape for the distributions of log prices; namely, they follow a Gaussiantype distribution with heavy tails. Moreover, we underline that the latter description, by analogy with the CB model, guarantees that the probability of percolation for the system is different from zero, where the percolation phenomenon, as shown in [15], is a characteristic of the interacting models that describe the stock market.

In the model proposed by Sornette and Zhou (see $[8,9])$, a variant of the Ising model is exploited, allowing each agent $i$ to interact only with his first neighbors $j$, and we write $i \sim j$. Such an interaction is described by buying, $\sigma_{i}=+1$, or by selling, $\sigma_{i}=-1$, a single asset. It follows that, in such a model, the decision of the agent $i$, at time $t \in \mathbb{N}^{+}$, is determined by the following rule:

$$
\sigma_{i}(t)=\operatorname{sign}\left[\sum_{j: i \sim j} K_{i}(t) \sigma_{j}(t)+s_{i} G(t)+\epsilon_{i}(t)\right],
$$

where three different information channels are taken into account, namely, mutual influence, $K_{i}$, external news, $s_{i} G$, and idiosyncratic judgment, $\epsilon_{i}$. In particular $K_{i}$ is taken as a function of time: namely,

$$
K_{i}(t)=b_{i}+\alpha K_{i}(t-1)+\beta r(t-1) G(t-1) .
$$

Therefore, it is impossible to define a potential related to (1) in statistical-mechanics terms. More precisely, according to [16], we note that the main characteristic of the Sornette and Zhou proposal, which is the main difference with the Ising model, concerns the fluctuation of the ratio $\mathrm{K} / \mathrm{s}$ which represents, following the authors, a measure of the tendency toward imitation relative to the tendency towards idiosyncratic behavior. In the following section, we provide a way to overcome this drawback by introducing a model that can be defined using a potential.

\section{The Model}

Let us start by considering a model with $N$ agents interacting in a network $\Gamma$, where $\Gamma$ is the set of nodes of the square lattice $\left(\Gamma \subset \mathbb{Z}^{2}\right)$, and suppose that each node interacts with the nearest neighbor and also with agents at a maximum distance of $\sqrt{2}$. We underline that such a description is chosen having in mind concrete implementations. Following the idea explained in [8], we suppose that, at each time step $t \in \mathbb{N}^{+}$, each agent $i \in \Gamma$ places a buy, a sell, and order, respectively, and hence, $\sigma_{i}(t)=-1$ and $\sigma_{i}(t)=+1$, respectively. The decision taken by a single agent is determined according to an interaction potential $\Phi$ that, roughly speaking, measures the value of the agent in each set $\Lambda \subset \Gamma$. Let us first consider the standard Ising approach, taking into account a potential

$$
\Phi_{\Lambda}= \begin{cases}K \sigma_{i} \sigma_{j}+h \sigma_{i}, & \Lambda=\left\{\sigma_{i}, \sigma_{j}\right\} \quad|i-j|=1 \\ 0, & \text { otherwise }\end{cases}
$$

where the interaction parameters $K, h$ in the Ising case are assumed to be constants, while, in what follows, we will consider them as functions of the node $i \in \Gamma$.

Considering (3) with respect to a Glauber dynamic, we have that the decision $\sigma_{i}(t)$ of the agent $i \in \Gamma$ at time $t \in \mathbb{N}^{+}$ is determined by the following rule:

$$
\sigma_{i}(t)=\operatorname{sign}\left[\beta\left(\sum_{j:|i-j|=1} K_{i} \sigma_{j}(t)+h_{i}\right)+\epsilon_{i}(t)\right] .
$$

In particular, (4) highlights the following three contributions:

(i) Imitation term: this is represented by the term $\sum_{j} K_{i} \sigma_{j}$, where $K_{i}$ is the relative propensity of the 
trader $i$ to be influenced by the sentiment of his nearest neighbors. We consider $K_{i}$ as a function constant in time, but not equal for all the agents. The latter assumption is due to the fact that we would give more importance to the dimension of the cluster around the agent $\sigma_{i}$ also taking into account how single agents are affected by such interaction, exploiting the idea that not all agents are affected in the same way. In our implementation $K_{i}$ is uniformly distributed in $\left[p_{\min }, p_{\max }\right]$, namely, $p_{i} \sim U\left(p_{\min }, p_{\max }\right)$ for every $i=1, \ldots, N$, while the constants $p_{\max }$ and $p_{\min }$ have been empirically determined as in [8]. To be precise the constants $p_{\min }$ and $p_{\max }$, respectively, define the minimum and the maximum, respectively, for the interval of validity of the probability distribution for the random quantity $p_{i}, i=1, \ldots, N$, according to the empirical tests performed with respect to the Sornette-Zhou constant $K$.

(ii) Impact of external news: this is represented by the term $h_{i}$, which represents the impact of external news and it is assumed to be constant in time, but we allow it to be not equal for each agent. In particular, $h_{i}$ is assumed to take value as a uniform random variable in the interval $\left(s_{\min }, s_{\max }\right)$, the constants $s_{\min }=-s_{\max }$ being determined empirically as in [8], even if in our case $s_{\max }$ describes the maximum concerning the news impact, while the susceptibility parameter, namely, the one indicated by $s$ in [8], has been replaced by the temperature.

(iii) Idiosyncratic judgment: this is represented by the term $\epsilon_{i}(t)$, which embodies the idiosyncratic content of the decision of agent $i$. It is being distributed following a Laplace distribution with mean 0 and variance 2 , as a result of the Gibbs sampling used to define our dynamics.

We suppose that the market price $p=p(t), t \in \mathbb{N}^{+}$, is updated according to

$$
p(t)=p(t-1) e^{r(t)},
$$

where we consider, as proposed by Wyart and Bouchaud in [17], that the return rate $r=r(t)$ is a linear function of the aggregate choosing of each agent at time $t$; in particular

$$
r(t)=\frac{\sum_{i \in \Gamma} \sigma_{i}(t)}{\lambda N^{2}},
$$

where the term $\lambda$ measures the liquidity of the market. In our analysis, according to real market data, we have to take $\lambda=40$. In the following we will consider the distribution of returns at different time scale $\tau$, and we write

$$
r_{\tau}(t):=\ln \left[\frac{p(t)}{p(t-\tau)}\right]
$$

where $\tau$ is taken as a multiple of time step.

As highlighted by the definition of the potentials (see (3) and (11)), our dynamics is characterized by the fact that the only evolving parameter is $\beta$, that is, the inverse of the temperature $T$. The latter idea, as previously mentioned, is mainly based on the approach proposed in $[10,11]$, where the authors used it to describe a stock market model. In particular, in [10], it is shown that, for short time lag, that is, $\tau=1$ minute, the distribution of the returns $f$ behaves according to

$$
f(r)=\frac{1}{2 T} \exp \left(\frac{|r|}{T}\right)
$$

where the parameter $T$, interpreted as the temperature of the market, is empirically calculated as $T=\sigma / \sqrt{2}, \sigma$ being the market volatility. It is worth mentioning that our model provides rather different results. In particular, we have phase transitions for low temperature, while, on the contrary, in [10] the probability of financial crashes is shown to grow when the temperature increases.

By analogy with the dynamics described in [8] for the parameters $K_{i}$, we define the following process for $\beta$ :

$$
\beta(t)=b+\alpha \beta(t-1)+r(t-1) G(t-1),
$$

which is analogous to the dynamic described in (2). In fact, miming [8], we account for the adaptive nature of agents and their learning abilities by updating the inverse of temperature. In particular, the parameter $b$ in (9) quantifies the intrinsic imitation influence of each agent in absence of other effects, and it is distributed uniformly in the interval $\left(0, b_{\max }\right)$, while the term $G(t)$ takes into account the impact that the external news term, namely, $I(t)$, has on the decision of agents. The latter term (see [8]) is assumed to be normally distributed, $I(t) \sim \mathcal{N}(0,1)$; therefore, $G(t)$ is determined by the following rule:

$$
G(t)= \begin{cases}1, & I(t) \geq 0 \\ -1, & I(t)<0\end{cases}
$$

It is worth mentioning that we multiply the third factor for a positive constant, that is, +1 , assuming that agents misattribute the origin of the market moves to herding rather than to impact of the news. The latter (see also [8]) confirms the idea, common to several economists, that the market follows an irrational behavior; see, for example, [18].

Following the example of Cont and Bouchaud in [5], and mainly for implementation and time consuming reasons, we consider a dynamic where agents act one at time, taking into account financial movements of the individuals that have preceded them.

Figure 1 represents the results obtained using the same values for the parameters as in [8]. In particular, Figure 1 shows that the distribution of log return follows the normal distribution; hence, we are not able to reproduce the desired heavy tails characteristics. Better results, in terms of adherence to real market data, cannot be obtained even considering parameters values far from those recommended by Sornette and Zhou, for example, taking $\alpha \leq 0.19$ or $s_{\max } \geq 0.22$, since, doing that, we obtain unrealistic distributions.

Nevertheless, noticing that in our proposal the accent is posed on the ratio $K / h$, instead of that on $K / s$ as in [8], we 


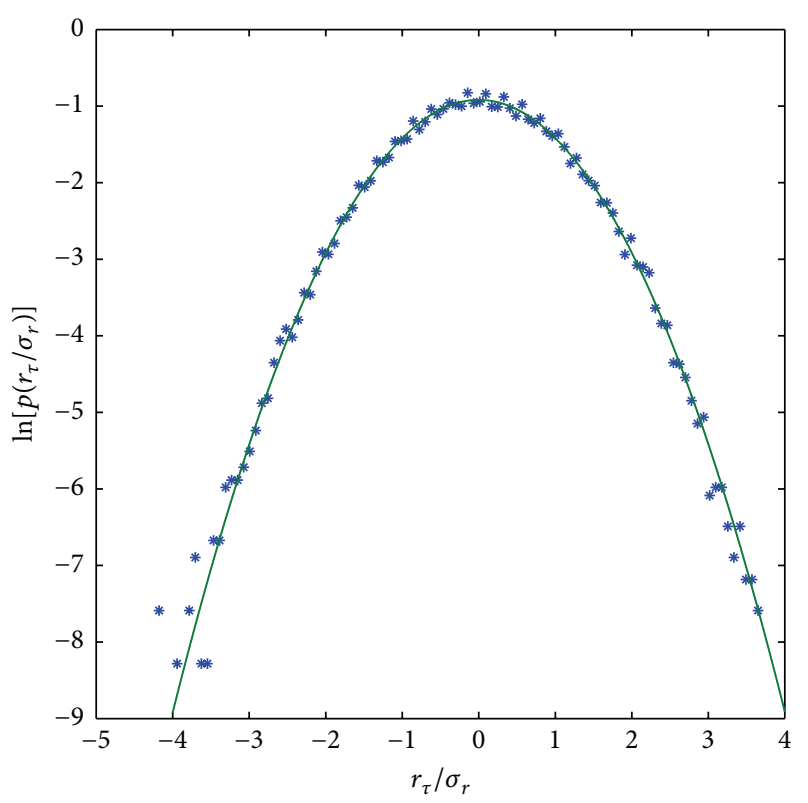

FIGURE 1: We compare the Gaussian distribution (solid line) with the pdf of log return with time scales $\tau=8$ (*-line) in the Ising case.

are led to changing the parameter related to the impact of external news, considering a new potential of the following form:

$$
\begin{aligned}
& \Phi_{\Lambda} \\
& =\left\{\begin{array}{lll}
K_{i} \sigma_{i} \sigma_{j}+\frac{h_{i}}{\sigma_{j}(1+\epsilon)+\sigma_{i}} \sigma_{i}, & \Lambda=\left\{\sigma_{i}, \sigma_{j}\right\} & |i-j|=1, \\
\frac{h_{i}}{\sigma_{j}(1+\epsilon)+\sigma_{i}} \sigma_{i}, & \Lambda=\left\{\sigma_{i}, \sigma_{j}\right\} & |i-j|=\sqrt{2},
\end{array}\right.
\end{aligned}
$$

where $\epsilon>0$ is a small quantity such that $\Phi_{\Lambda}$ in (11) is still a $C$ potential. Therefore, as in the Ising case, there exists a critical inverse temperature $\beta_{C}$ such that the system undergoes phase transition, with ground states: $\{(+-;+-),(-+;-+)\}$. Now, hiding the time dependence, we define the Hamiltonian function as

$$
H=\sum_{i \in \Gamma} K_{i} \sum_{j:|i-j|=1} \sigma_{i} \sigma_{j}+4 \sum_{i \in \Gamma} h_{i} \sum_{j:|i-j| \leq \sqrt{2}} \frac{\sigma_{j}}{\sigma_{i}+\sigma_{j}(1+\epsilon)},
$$

and then, using the Glauber dynamics approach, we obtain the following rule of advancement:

$$
\begin{aligned}
& \sigma_{i}(t)=\operatorname{sign}[\beta(t) \\
& \quad\left(\sum_{j:|i-j|=1} K_{i} \sigma_{j}(t)+\tilde{h}_{i} \sum_{j:|i-j| \leq \sqrt{2}} \sigma_{i} \frac{1+\epsilon}{\sigma_{j}(t)\left[\epsilon^{2}+2 \epsilon\right]}\right) \\
& \left.+\epsilon_{i}(t)\right] .
\end{aligned}
$$

We would like to underline that our approach is characterized by changing only the impact of external news term; in particular, in this new form, the external factor is not constant, but it is strongly dependent on the external values, while the $\beta$-dynamic remains unchanged. Moreover, in our model, the parameter $h$ represents the idiosyncratic susceptibility of each agent, while the impact effect due to external news is implicitly given by the nearest agents; namely, the news is provided by the agents themselves, as pointed out in [5]. The next section illustrates the results obtained according to (11) and (13).

\section{Simulations and Results}

In our simulations we consider a square lattice with $50 \times 50=$ $N$ nodes, where each agent $i$, taking decisions $\sigma_{i}(t), t \in \mathbb{N}^{+}$, represents a node of the lattice and it is allowed to interact with the nearest neighbors and with agents at $\sqrt{2}$ distance, as highlighted in (11). According to the previously mentioned rule of taking decisions one at a time (see $[5,8]$ ), we are left with the definition of an ordered process with maturity time $T=5 \times 10^{4}$ time steps. We consider $\widetilde{h}_{i}$ as a parameter constant in time but not equal for all agents. In particular, the latter parameter is taken as a combination of two functions: $\widetilde{h}_{i}=$ $s_{i} g$, where $s_{i}$, defined at the beginning of the simulation and frozen thereafter, is uniformly distributed in $\left(0, s_{\max }\right)$, while $g$ is a universal constant. Our parameters choice has been made according to the following scheme: we start with values as in the SZ-model; then, through an optimization procedure based on iterative simulations, we determine the final values. The latter procedure allows us to have a maximum value, that is, $s_{\max }$, which is half of the one obtained in [8], where the authors consider a 4-neighbor interaction, while we deal with an 8-neighbor one.

The simulation of the latter model, as described by (13), presents some difficulties, especially concerning the numerical implementation of the second term. Therefore, by algebraic manipulation and assuming a reasonable ansatz, we define an operative form of this rule of advancement. In particular,

$$
\sum_{j:|i-j| \leq \sqrt{2}} \frac{1+\epsilon}{\sigma_{j}(t)\left[\epsilon^{2}+2 \epsilon\right]}
$$

is equivalent to

$$
(p-n) \frac{1+\epsilon}{\epsilon(2+\epsilon)},
$$

where $p, n \in \mathbb{N}$ are, respectively, the number of neighbors of agent $i$, taking decisions +1 and -1 . Because agents are allowed to take decisions one at a time, taking into account the decisions of the individuals preceding them, we have that a good approximation of parameter is given by the inverse of $r(t) G(t)$, which is then the one used in our implementations. Moreover, during calculations, the latter quantity is increased by a small constant, $\delta \geq 0$, which guarantees that the second coefficient remains bounded; namely, it takes the place of $\epsilon$ in (15).

Even if we have tested several combinations and values for the involved parameters, for the sake of clarity, we limit 


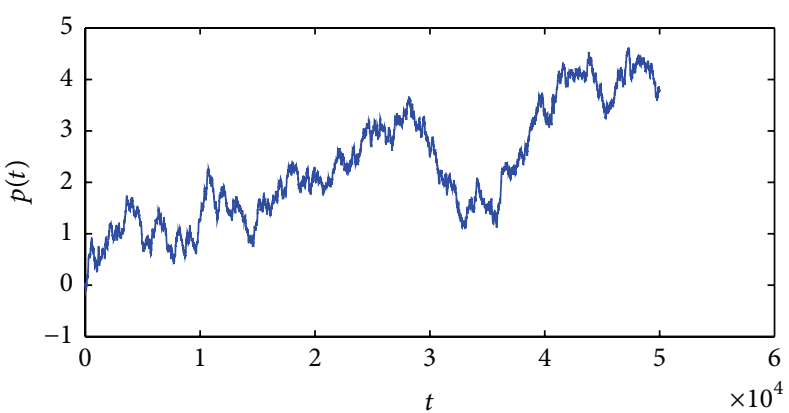

(a)

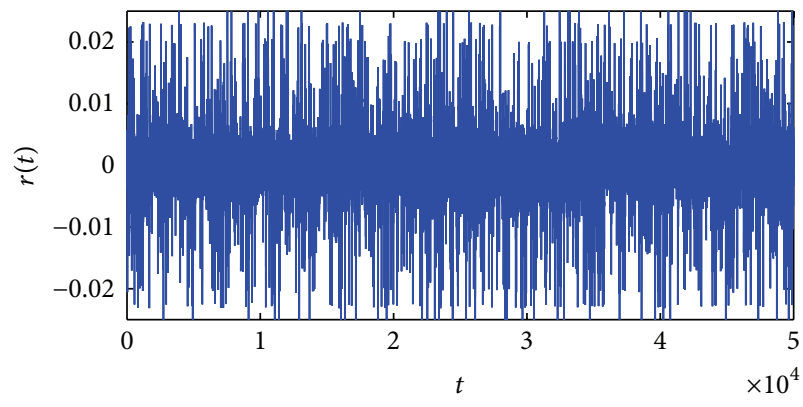

(b)

Figure 2: In these figures we show the realization of the logarithm of the price over $5 \times 10^{4}$ time steps (a), and the time series of the log returns of the price (b).

ourselves to showing the results obtained for the following two sets of specifications, mostly because they are the most relevant ones. In particular, we consider

$$
\begin{aligned}
& \left(\alpha ; \delta ; b_{\max } ; g ; s_{\max } ; p_{\min } ; p_{\max }\right) \\
& \quad=\left(0.20 ; 0 ; 1 ; \frac{1}{101} ; 0.15 ; 0 ; 20\right),
\end{aligned}
$$

and, taking $K=1, s=0.03$ as constants for each agent, the following vector of values:

$$
\left(\alpha ; \delta ; b_{\max } ; g\right)=\left(0.21 ; 0.00208 ; 1 ; \frac{1}{45}\right) .
$$

Note that the choice $K=1$ implies that we do not have to take into account the values of $p_{\min }$ and $p_{\max }$.

It is worth mentioning that the latter combinations of parameters are representative of a rather large range. In particular, following the approach used in $[5,8]$, and references therein, the intervals of validity for the parameters appearing in the first combination are $\alpha \in(0.21,0.22), \delta \leq 10^{-10}$, and $g \in(1 / 99,1 / 105)$, while for the second set we have $\delta \in(0.002,0.00213)$ and $g \in(1 / 43,1 / 45)$.

Remark 1. As an example concerning the aforementioned ranges for parameters values, we have performed a large set of computations observing that we are obliged to choose $\alpha \geq$ 0.195 to avoid the unrealistic phenomenon of bimodal distribution. From an econophysic point of view, the latter implies that the temperature of the market does not lose sufficiently quickly the memory of the past (financial) actions made by agents acting within the market, experiencing, instead, a slow evolution. From an economic point of view previous phenomenon means (see [8]) that the model explores the ordered regime of the Ising model too often; namely, agents form a crowd with a majority opinion which can flip in time \pm 1 .

\subsection{Probability Density Functions of Log Returns at Different} Time Scales. According to the first specification of parameters (see (16)), Figure 2 provides a graphical representation of the distribution of the logarithm of prices over time, clearly showing, by analogy with real market data, the phenomenon

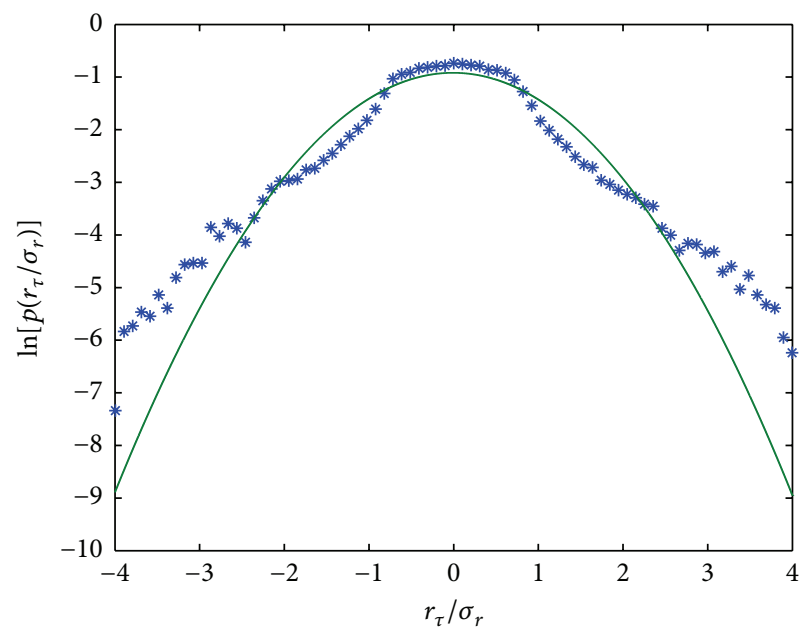

FIGURE 3: The figure shows a comparison between the empirical distribution of the log returns, considering $\tau=8$ (*-line), and the Gaussian standard distribution.



Figure 4: Empirical pdf of log return with $\tau=32$ (*-line), and theoretical distribution, Gaussian (solid line).

of volatility clustering. In fact large changes in prices tend to cluster together, as highlighted, for example, in [19].

In Figures 3 and 4, using the semilogarithmic scale, we compare the sample distribution of the log returns of our model plotted with the star, while with the solid line we represent the normal distribution. 


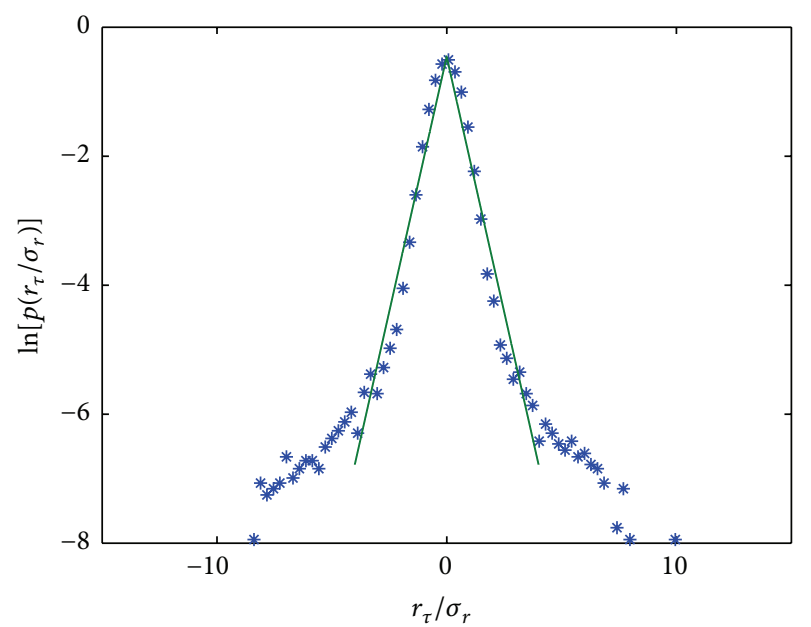

FIgURE 5: Distribution of returns with $\tau=16(*$-line) in the case $K=$ const, and Laplace distribution (solid line).

We note that Figure 3, where the time lag equals $\tau=8$, clearly shows the presence of the fat tails, hence capturing one of the main characteristics of real financial time series.

Figure 4 shows that, by analogy with well-established results in the literature (see, e.g., [20]), when the time scale equals $\tau=32$, the distribution of $\log$ return is Gaussian.

Using the second specification of parameters (see (17)), we obtain quite different results. In fact, as we see in Figure 5 the distribution of $r_{\tau}$, for $\tau=16$, is of Laplace-type, with only a very small set of rare events, displaying heavy tails, which do not follow the Laplace distribution. This characteristic has been highlighted in [10] in the case of time lag of one-minute length. The latter results imply that if we change the value of some parameters, we are able to model rather different market scenarios. In particular, we can also observe the presence of volatility clustering (see Figure 6), which is a highly desirable feature and also another proof of our model's goodness.

4.2. Autocorrelations. Figures 7 and 8 show the temporal correlation of the $\log$ return $r$, as defined in Figure 5, as a function of time lag $\tau$. In particular, Figure 7 is associated with the first specification of parameters (see (16)), and it constitutes the basis for what is shown in Figure 3, while Figure 8 is associated with the specification given in (17), which provides the distribution shown in Figure 5.

It is worth mentioning that in both cases a very short correlation time is revealed, namely, of duration smaller than one step. We underline that this behavior agrees with the stylized facts on the correlation of returns and of volatility of real financial markets. In particular, as shown in [3], empirically results highlight short-range correlations in price changes with memory time which is less than one trading day. Exploiting latter observations and comparing our results with real market distributions, we can observe that, for time scales $\tau=4$ and $\tau=8$, the distributions of the returns of the first data set (16) are similar to the empirical one daily scale; therefore, it is reasonable to approximate $4-8$ time steps with one day. Instead, taking into account data set (17), we have that 8-16 time steps $\tau$ approximately correspond to 1 minute.

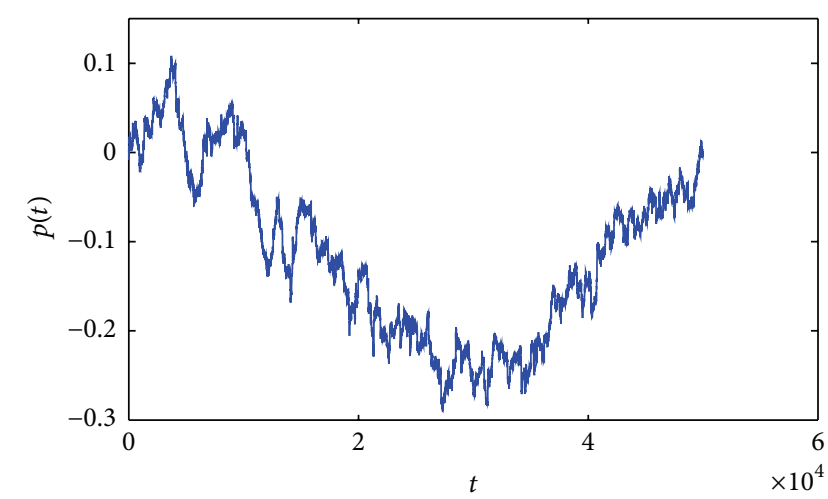

FIgURE 6: Realization of the logarithm of the prices, according to parameters specifications (17).



Figure 7: Autocorrelation function of the log returns of the realization shown in Figure 3.

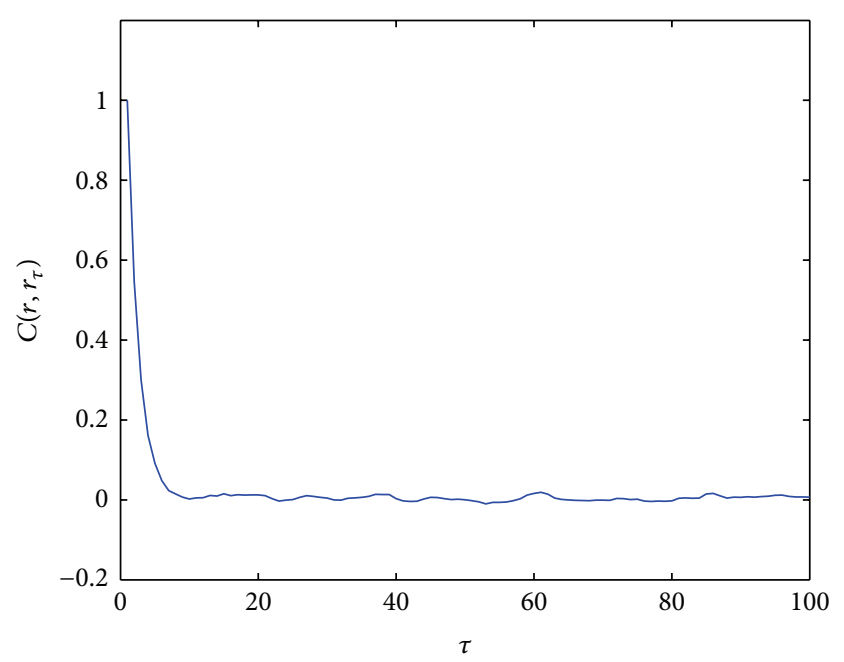

FIGURE 8: Autocorrelation function of the log return of the realization shown in Figure 5. 
Previous results suggest that if we rewrite the potential of the first simulation (see (11)), in the light of the potential used in the second simulation, namely, (11) with $K$ constant, we obtain that the temperature that changes its value at each node is becoming a function of the space and the time. Therefore, taking the inverse of the temperature, namely, the parameter $\beta$, we have that $\beta=\beta(i, t)$, with $(i, t) \in \Gamma \times \mathbb{N}^{+}$. It is important to note that the latter interpretation is very close to the ideas developed in $[5,19,21]$, allowing us to establish a close relation between the clusters dimension and the inverse of temperature; namely, they are positively related.

\section{Conclusions}

In the present paper we have taken into account a modification of the Ising model, changing the external field term to obtain interesting econophysics results, without losing the good properties of the classic model. In particular, we have shown how the probability density functions characterizing both the log returns and the intracorrelation of them are in agreement with the results obtained in $[8,9,22]$ and with the ones forecasted in $[5,19,21]$. The latter implies that our analysis agrees with the empirical distributions of real market time series.

Our model shows that the temperature can be considered constant throughout the stylized financial network, thought of as network of interconnected and interacting financial agents, if and only if we consider very short time lag. In particular, we can conclude that if we want to work with realistic time scales, we have to consider different temperatures acting at different nodes; namely, each agent in the network has to be characterized by its proper temperature parameter.

We would like to underline that extensions of the presented approach will be the subject of our future works. In particular, we aim to relax the assumption that each agent interacts only with his nearest neighbors, by considering a possibly finite range, but with interaction length greater than $\sqrt{2}$, potential.

It is worth mentioning that the aforementioned types of potentials are often used in statistical-mechanics applications, hence allowing us to exploit known results to better understand those collective phenomena which steer real financial networks.

\section{Conflict of Interests}

The authors declare that they have no conflict of interests regarding the publication of this paper.

\section{References}

[1] T. Lux, "The socio-economic dynamics of speculative markets: interacting agents, chaos, and the fat tails of return distributions," Journal of Economic Behavior and Organization, vol. 33, no. 2, pp. 143-165, 1998.

[2] J. T. MacHado, F. B. Duarte, and G. M. Duarte, "Power law analysis of financial index dynamics," Discrete Dynamics in Nature and Society, vol. 2012, Article ID 120518, 12 pages, 2012.
[3] R. N. Mantegna and H. E. Stanley, An Introduction to Econophysics: Correlations and Complexity in Finance, Cambridge University Press, Cambridge, UK, 2000.

[4] Y.-T. Bian, L. Xu, J.-S. Li, J.-M. He, and Y.-M. Zhuang, "An evolution model of trading behavior based on peer effect in networks," Discrete Dynamics in Nature and Society, vol. 2012, Article ID 138178, 15 pages, 2012.

[5] R. Cont and J.-P. Bouchaud, "Herd behavior and aggregate fluctuations in financial markets," Macroeconomic Dynamics, vol. 4, no. 2, pp. 170-196, 2000.

[6] D. S. Scharfstein and J. C. Stein, "Herd behavior and investment," American Economic Review, vol. 80, no. 3, pp. 465-479, 1990.

[7] E. Callen and D. Shapero, "A theory of social imitation," Physics Today, vol. 27, no. 7, p. 23, 1974.

[8] D. Sornette and W.-X. Zhou, "Importance of positive feedbacks and overconfidence in a self-fulfilling Ising model of financial markets," Physica A: Statistical Mechanics and Its Applications, vol. 370, no. 2, pp. 704-726, 2006.

[9] W.-X. Zhou and D. Sornette, "Self-organizing Ising model of financial markets," The European Physical Journal B, vol. 55, no. 2, pp. 175-181, 2007.

[10] H. Kleinert and X. J. Chen, "Boltzmann distribution and market temperature," Physica A, vol. 383, no. 2, pp. 513-518, 2007.

[11] A. Dragulescu and V. M. Yakovenko, "Statistical mechanics of money," The European Physical Journal B-Condensed Matter and Complex Systems, vol. 17, no. 4, pp. 723-729, 2000.

[12] P. Erdös and A. Rényi, "On random graphs. I," Publicationes Mathematicae Debrecen, vol. 6, pp. 290-297, 1959.

[13] E. N. Gilbert, "Random graphs," Annals of Mathematical Statistics, vol. 30, no. 4, pp. 1141-1144, 1959.

[14] P. A. Ferrari, E. A. Pechersky, V. V. Sisko, and A. A. Yambartsev, "Gibbs random graphs on point processes," Journal of Mathematical Physics, vol. 51, no. 11, Article ID 113303, 2010.

[15] A. Pei and J. Wang, "Nonlinear analysis of return time series model by oriented percolation dynamic system," Abstract and Applied Analysis, vol. 2013, Article ID 612738, 12 pages, 2013.

[16] A. Johansen, D. Sornette, and O. Ledoit, "Predicting financial crashes using discrete scale invariance," Journal of Risk, vol. 1, no. 4, pp. 5-32, 1999.

[17] M. Wyart and J.-P. Bouchaud, "Self-referential behaviour, overreaction and conventions in financial markets," Journal of Economic Behavior and Organization, vol. 63, no. 1, pp. 1-24, 2007.

[18] E. F. Fama, "The behavior of stock market price," The Journal of Business, vol. 38, no. 1, pp. 34-105, 1965.

[19] R. Cont, "Volatility clustering in financial markets: empirical facts and agent-based models," in Long Memory in Economics, pp. 289-309, Springer, Berlin, Germany, 2007.

[20] S. Ghashghaie, W. Breymann, J. Peinke, P. Talkner, and Y. Dodge, "Turbulent cascades in foreign exchange markets," Nature, vol. 381, no. 6585, pp. 767-770, 1996.

[21] R. Cont, M. Potters, and J.-P. Bouchaud, "Scaling in stock market data: stable laws and beyond," in Scale Invariance and Beyond, B. Dubrulle, F. Graner, and D. Sornette, Eds., vol. 7 of Centre de Physique des Houches, pp. 75-85, Springer, 1997.

[22] D. Stauffer and D. Sornette, "Self-organized percolation model for stock market fluctuations," Physica A: Statistical Mechanics and Its Applications, vol. 271, no. 3-4, pp. 496-506, 1999. 


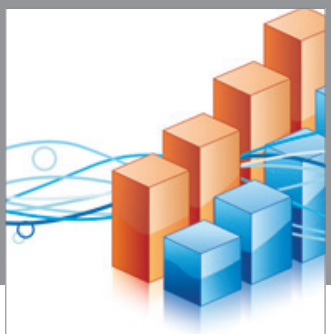

Advances in

Operations Research

vatem alat4

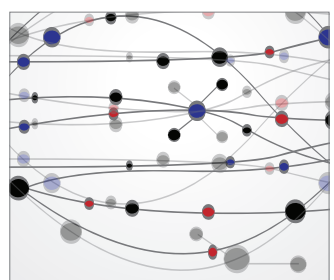

\section{The Scientific} World Journal
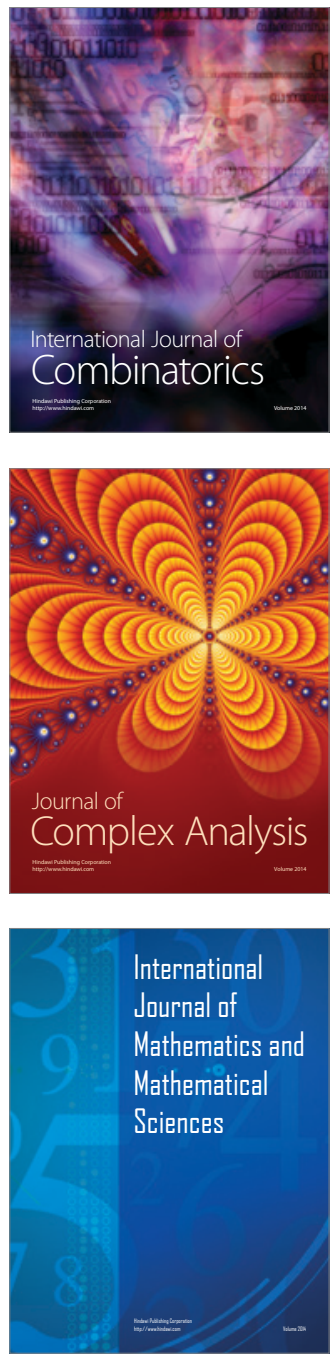
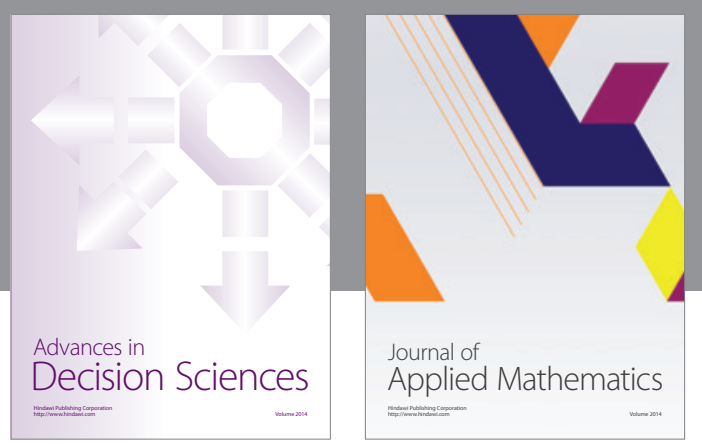

Algebra

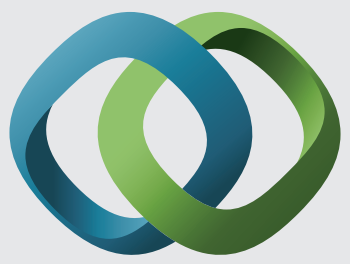

\section{Hindawi}

Submit your manuscripts at

http://www.hindawi.com
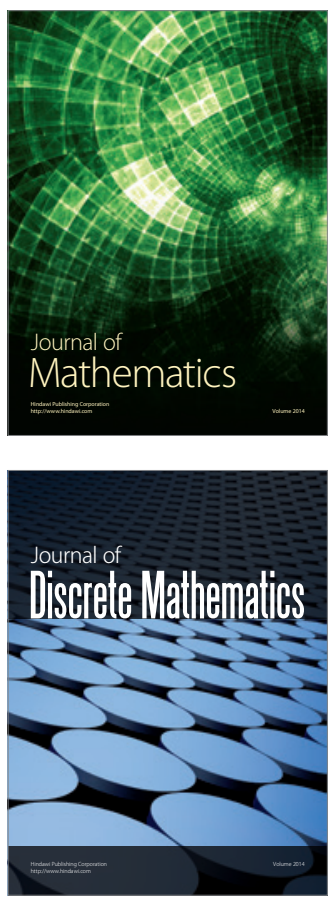

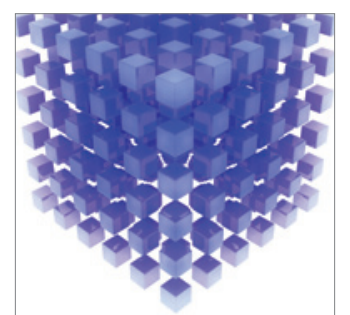

Mathematical Problems in Engineering
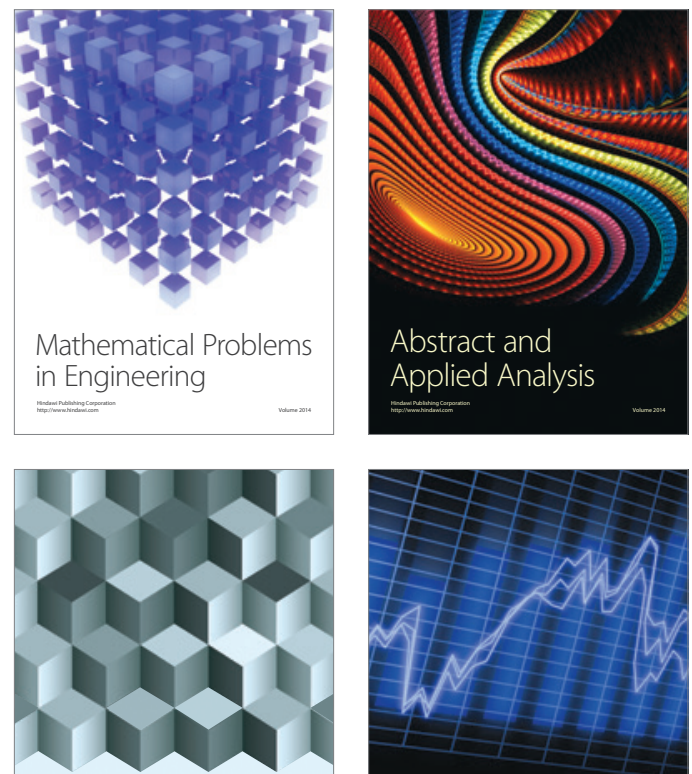

Journal of

Function Spaces

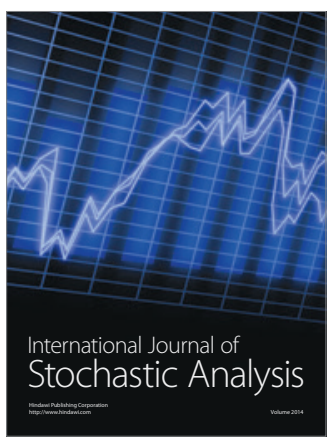

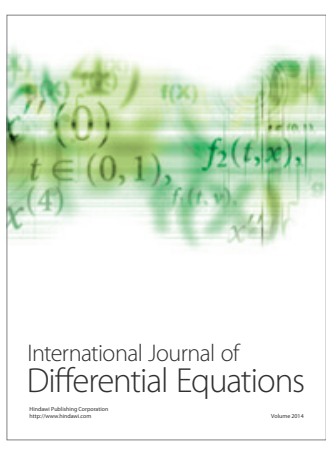
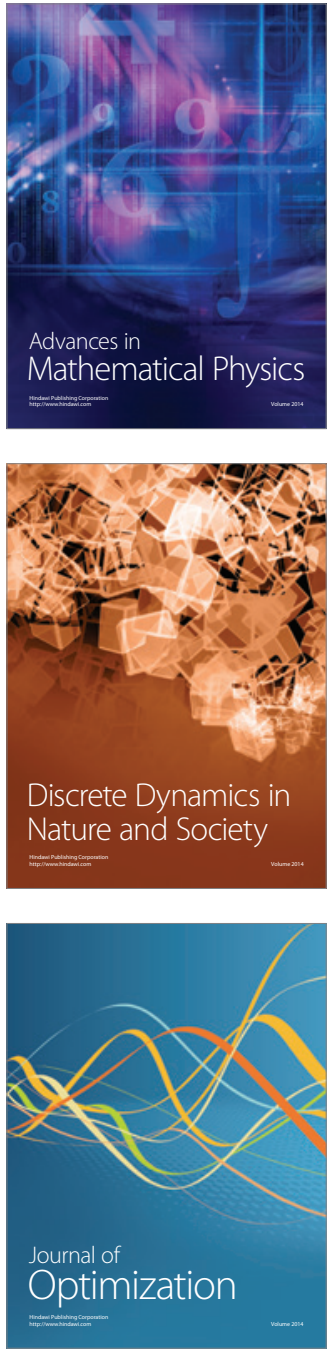